\title{
Numerical Solution of Two Transcendental Equations*
}

\author{
By Luciano Misici
}

\begin{abstract}
This paper deals with the study of the transcendental equations: $\sin (s+\nu) /(s+\nu)$ $= \pm \sin (s-\nu) /(s-\nu)$, where $\nu=\left(s^{2}-\gamma^{2}\right)^{1 / 2}$. These equations are obtained in the study of some boundary value problems for a modified biharmonic equation using the PapkovichFadle series. Some numerical solutions obtained with an iterative procedure are given.
\end{abstract}

Introduction. It is well known that the governing equation of a bidimensional Stokes flow parallel to the $x, y$ plane, in terms of the stream function $\Psi(x, y)$, is the biharmonic equation

$$
\nabla^{4} \Psi=\frac{\partial^{4} \Psi}{\partial x^{4}}+\frac{\partial^{4} \Psi}{\partial x^{2} \partial y^{2}}+\frac{\partial^{4} \Psi}{\partial y^{4}}=0 .
$$

The solution of Eq. (1) in a rectangle with homogeneous conditions on $\Psi$ and on its normal derivative on two parallel sides and for sufficiently regular boundary conditions on the remaining two parallel sides is expressed using the PapkovichFadle (PF) series. The even and odd eigenfunctions are associated to the roots, in the complex plane, of the equations

$$
\begin{aligned}
& \sin 2 s=-2 s, \\
& \sin 2 s=2 s .
\end{aligned}
$$

A complete study of Eqs. (2a) and (2b) may be found in [1]. Another interesting case is represented by the study of the equation

$$
\nabla^{4} \Psi-\gamma^{2} \nabla^{2} \Psi=0
$$

where $\nabla^{2}=\partial^{2} / \partial x^{2}+\partial^{2} / \partial y^{2}$ and $\gamma$ is real.

Equation (3) occurs, for example, in the study of the fluid motion in porous media [2] when Brinkman's approximation [3], [4] is used. The same equation has been used by de Socio, Gaffuri and the author [5] in the study of a tridimensional Stokes flow. If the solution of Eq. (3) is expressed in terms of PF series, the equations corresponding to $(2 a),(2 b)$ are

$$
\begin{aligned}
& \sin (s+\nu) /(s+\nu)=-\sin (s-\nu) /(s-\nu), \\
& \sin (s+\nu) /(s+\nu)=\sin (s-\nu) /(s-\nu),
\end{aligned}
$$

where $\nu=\left(s^{2}-\gamma^{2}\right)^{1 / 2}$. This note deals with a numerical study of Eqs. (4a), (4b) and gives a method to find all the real and complex solutions for any real value of $\gamma$.

Received March 29, 1983.

1980 Mathematics Subject Classification. Primary 65H05.

*This work was partially supported by Ministero della Pubblica Istruzione under contract $60 \%$, $81 / 00806$ cap 03. 
1. Analysis. All the roots of Eqs. (2a) and (2b) are complex except for $s=0$. We just notice that, for Eqs. (2a), (2b) and (4a), (4b), if $s$ is a complex root, then $-s$ and $\bar{s}$ are also roots. This study deals only with the roots in the first quadrant of the complex plane. Let $s_{n}=x_{n}+i y_{n}$ be the complex roots of Eqs. (2a), (2b) and (4a), (4b) ordered for $\operatorname{Re}\left(s_{n}\right)$ increasing taking into account the appropriate multiplicity. An asymptotic [6] evaluation of $s_{n}$ is

$$
s_{n}=\left(n \mp \frac{1}{4}\right) \pi+\frac{i}{2} \ln [(4 n \mp 1) \pi], \quad n=1,2, \ldots,
$$

for the two cases (2a) and (2b), respectively. The expression (1.1) gives an excellent initial guess for finding the roots of Eqs. (2a), (2b) through an iterative procedure. Since when $\gamma \rightarrow 0$ Eqs. (4a), (4b) approach Eqs. (2a), (2b), for $\gamma$ sufficiently small the values (1.1) are good initial guesses for the roots of $(4 a)$ and (4b). Some problems arise when $\gamma$ is large. In this case both (4a) and (4b) have real roots different from $s=0$ and $s= \pm \gamma$. In the following we study the real positive roots different from $s=0$ or $s= \pm \gamma$.

Case $4 \mathrm{a}$. In this case it is easy to see that the real positive roots of $(4 \mathrm{a})$ correspond to the zeros of the real function $f(x)$ of the real variable $x$ given by:

$$
f_{\gamma}(x)=\left\{\begin{array}{rlrl}
\bar{f}_{\gamma}(x) \equiv & x \sin x \cosh \sqrt{\gamma^{2}-x^{2}} & \\
& +\sqrt{\gamma^{2}-x^{2}} \cos x \sinh \sqrt{\gamma^{2}-x^{2}}, \quad 0 \leqslant x \leqslant \gamma, \\
f_{\gamma}^{*}(x) \equiv & x \sin x \cos \sqrt{x^{2}-\gamma^{2}} \\
& -\sqrt{x^{2}-\gamma^{2}} \cos x \sin \sqrt{x^{2}-\gamma^{2}}, \quad x>\gamma .
\end{array}\right.
$$

From the study of $f(x)$ one can see that there exists a value $\gamma_{1}^{*}$ below which the function does not have any zeros, whereas, for $\gamma=\gamma_{1}^{*}, f(x)$ presents a double zero. Let $\gamma \in[0, \infty)$. It will be shown that there is an increasing sequence $\left\{\gamma_{n}^{*}\right\}_{n=1}^{\infty}$ such that the total number of the real positive zeros of $f$ is given by:

$$
\begin{aligned}
& \left.\begin{array}{ll}
2(n-1) & \text { simple roots } \\
1 & \text { double root }
\end{array}\right\} \quad \text { for } \gamma=\gamma_{n}^{*}, \\
& 2 n \text { simple roots for } \gamma_{n}^{*}<\gamma<\gamma_{n+1}^{*} \text {. }
\end{aligned}
$$

The relevant value $\gamma_{n}^{*}$ may be obtained by solving the nonlinear system

$$
\left\{\begin{array}{l}
F(x, \gamma)=0, \\
\frac{\partial F(x, \gamma)}{\partial x}=0
\end{array}\right.
$$

where $F(x, \gamma) \equiv \bar{f}_{\gamma}(x)$.

Case $4 \mathrm{~b}$. As in the case $(4 \mathrm{a})$, the real positive roots correspond to the zeros of the function:

$$
g_{\gamma}(x)=\left\{\begin{array}{rlr}
\bar{g}_{\gamma}(x) \equiv \sqrt{\gamma^{2}-x^{2}} \sin x \cosh \sqrt{\gamma^{2}-x^{2}} & \\
& -x \cos x \sinh \sqrt{\gamma^{2}-x^{2}}, & 0 \leqslant x \leqslant \gamma, \\
g_{\gamma}^{*}(x) \equiv & \sqrt{x^{2}-\gamma^{2}} \sin x \cos \sqrt{x^{2}-\gamma^{2}} & \\
& -x \cos x \sin \sqrt{\gamma^{2}-x^{2}}, & x>\gamma .
\end{array}\right.
$$


Also, in this case, the numerical solution shows that there is an increasing sequence $\left\{\hat{\gamma}_{n}\right\}_{n=1}^{\infty}$ of values of $\gamma$ such that the root $x=\gamma$, which in general is simple, has multiplicity two. As before the total number of the positive real roots for (4b) increases with increasing $\gamma$ following (1.2). The values $\hat{\gamma}_{n}$ may be simply obtained by computing the positive real roots of the function

$$
G(\gamma)=\gamma \operatorname{cotg} \gamma-1
$$

2. Numerical Results. The real positive roots of $f(x)$ can be found as the intersections between the functions

$$
\begin{aligned}
& \begin{cases}h_{1}(x)=-x \operatorname{tg} x & \text { for } 0 \leqslant x \leqslant \gamma, \\
h_{2}(x)=\sqrt{\gamma^{2}-x^{2}} \operatorname{tgh} \sqrt{\gamma^{2}-x^{2}} & \end{cases} \\
& \left\{\begin{array}{l}
h_{3}(x)=x \operatorname{tg} x \\
h_{4}(x)=\sqrt{x^{2}-\gamma^{2}} \operatorname{tg} \sqrt{x^{2}-\gamma^{2}} \quad \text { for } x>\gamma .
\end{array}\right.
\end{aligned}
$$

From Figure 1, since $h_{1}(x)$ is independent of $\gamma$, we have that the critical value $\gamma_{n}^{*}$ of $\gamma$ occurs when $h_{1}(x)$ is tangent to $h_{2}(x)$. Moreover, for $\gamma=\bar{\gamma}$ the following intersections are obtained:

$$
\begin{array}{lll}
n-1 & \text { for } & (2 n-1) \frac{\pi}{2}<\bar{\gamma}<\gamma_{n}^{*} \\
n+1 & \text { for } & \gamma_{n}^{*} \leqslant \bar{\gamma} \leqslant n \pi \\
n & \text { for } & n \pi<\bar{\gamma} \leqslant(2 n+1) \frac{\pi}{2}
\end{array}
$$

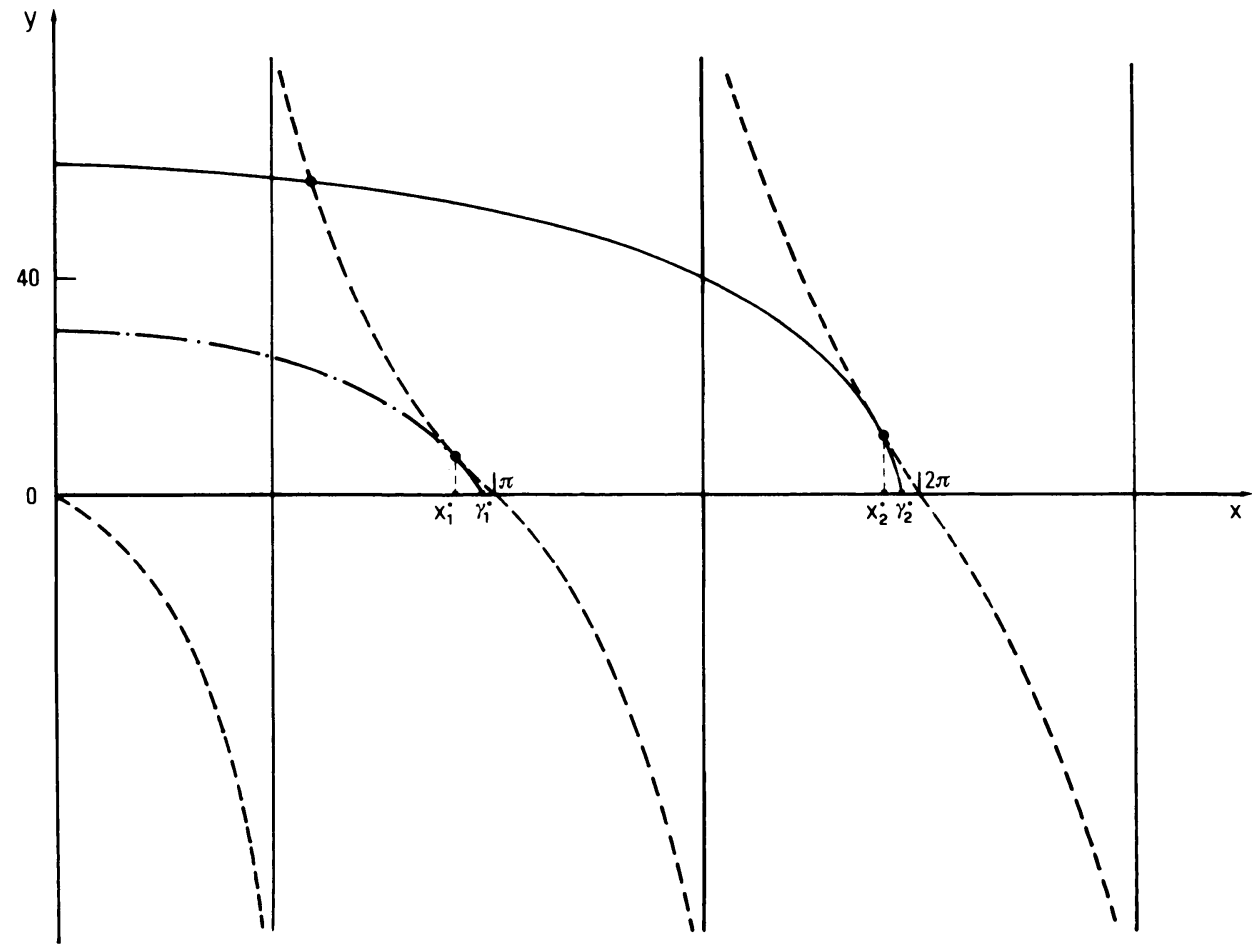

FIGURE 1

Qualitative behavior of: $h_{1}(x),(----) ; h_{2}(x)$ for $\gamma=\gamma_{1}^{*}(-.-.-)$

and $\gamma=\gamma_{2}^{*}(-)$. 
TABLE 1

\begin{tabular}{|c|c|c|c|}
\hline $\mathrm{n}$ & $\gamma_{n}^{*}$ & $x_{n}^{\star}$ & $\dot{\hat{\gamma}}_{n}$ \\
\hline 1 & 3.044699213 & 2.798386046 & 4.493409458 \\
\hline 2 & 6.237702792 & 6.121250467 & 7.725251837 \\
\hline 3 & 9.394778563 & 9.317866462 & 10.904121659 \\
\hline 4 & 12.543953611 & 12.486454395 & 14.066193913 \\
\hline 5 & 15.690059952 & 15.644128370 & 17.220755272 \\
\hline 6 & 18.834650141 & 18.796404366 & 20.371302959 \\
\hline 7 & 21.978379228 & 21.945612880 & 23.519452499 \\
\hline 8 & 25.121572041 & 25.092910412 & 26.666054259 \\
\hline 9 & 28.264408144 & 28.238936575 & 29.811598791 \\
\hline 10 & 31.406994934 & 31.384074018 & 32.956389039 \\
\hline 11 & 34.549400602 & 34.528565755 & 36.100622244 \\
\hline 12 & 37.691670537 & $37 \cdot 672573565$ & $39 \cdot 244432361$ \\
\hline 13 & 40.833836124 & 40.816209326 & 42.387913568 \\
\hline 14 & 43.975919763 & 43.959552889 & 45.531134014 \\
\hline 15 & 47.117937867 & 47.102662770 & 48.674144232 \\
\hline 16 & 50.259902741 & 50.245582838 & 51.816982487 \\
\hline 17 & 53.401823788 & 53.388346622 & 54.959678288 \\
\hline 18 & 56.543708319 & 56.530980194 & 58.102254754 \\
\hline 19 & 59.685562107 & 59.673504130 & 61.244730260 \\
\hline 20 & 62.827389766 & 62.815934889 & 64.387119591 \\
\hline
\end{tabular}

Analogously it has been observed that a branch of $h_{4}(x)$ intersects a branch of $h_{3}(x)$ only if the zero of $h_{4}(x)$ is greater than the one of $h_{3}(x)$ and if the asymptote at the right of the first is smaller than the one at the right of the second. The converse is true if the left asymptote is considered. The number of intersections in this case is:

$$
\begin{array}{ll}
n-1 \quad \text { for } \quad(2 n-1) \frac{\pi}{2}<\bar{\gamma} \leqslant n \pi, \\
n \quad \text { for } n \pi<\bar{\gamma} \leqslant(2 n+1) \frac{\pi}{2} .
\end{array}
$$




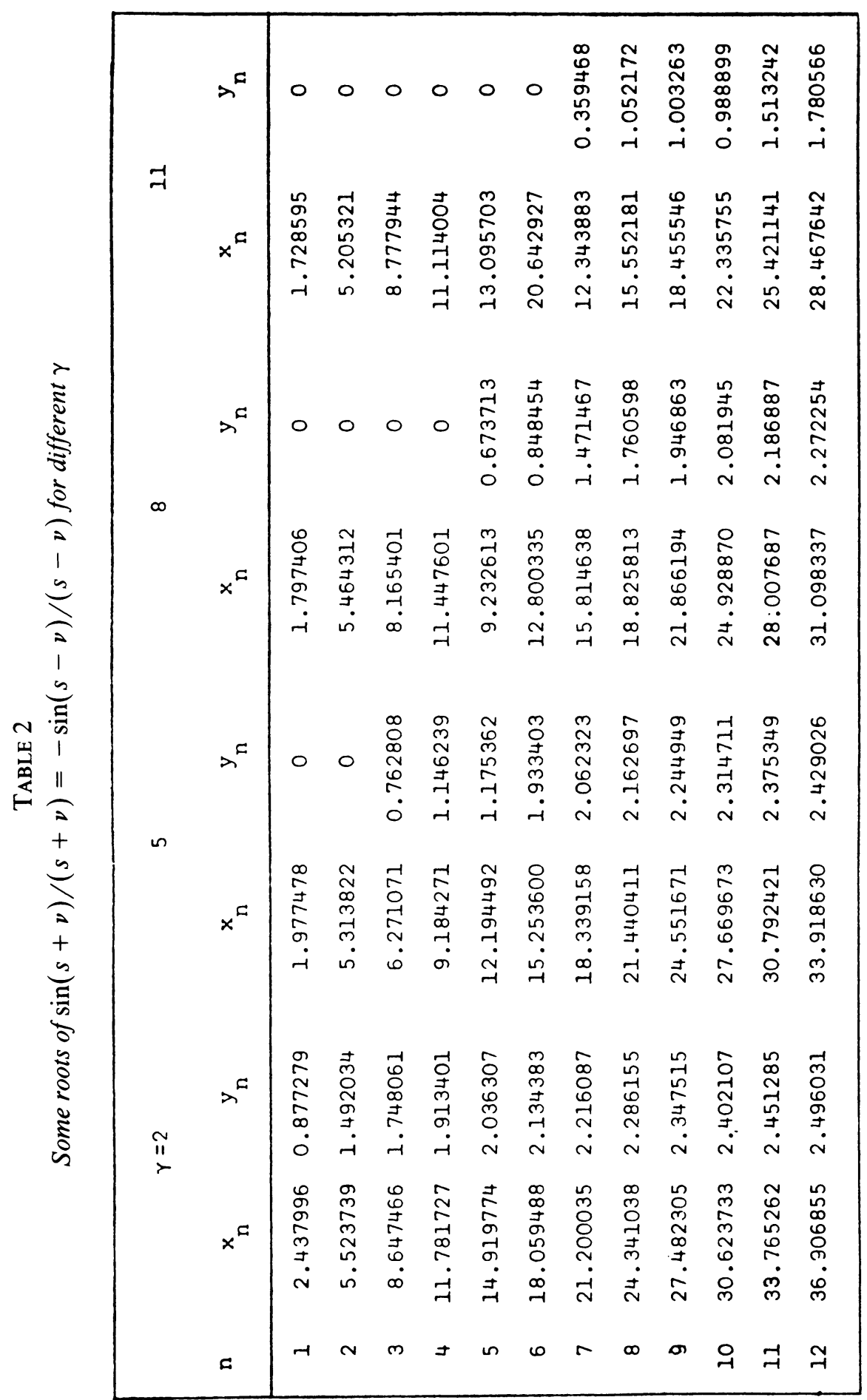




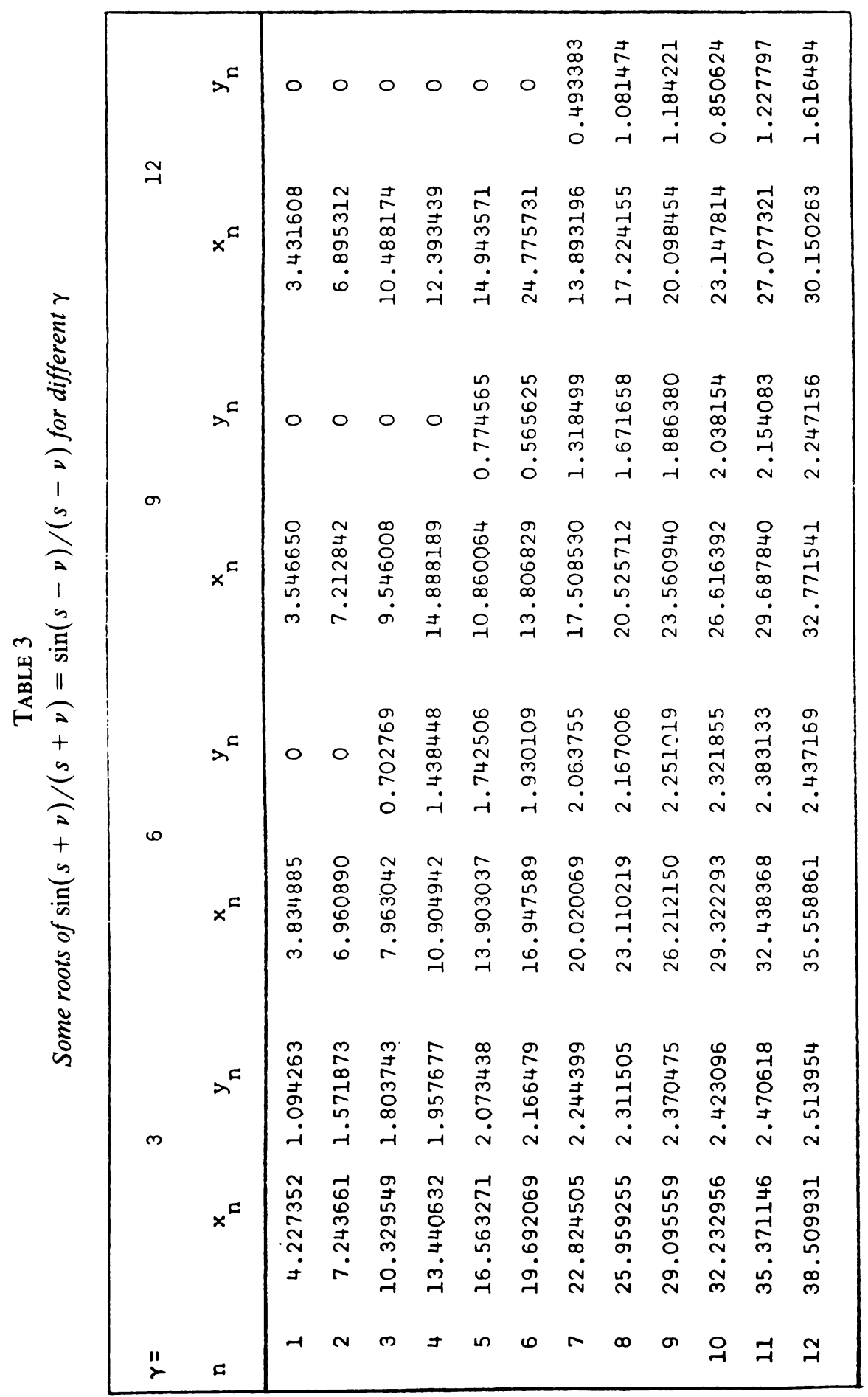


One can note that in this case the relation (1.2) is verified and the values $\gamma_{n}^{*}$ are obtained by (1.3). Similar considerations are valid for the case (4b). With such a study it is possible to isolate all the roots of (4a) and (4b), and then to obtain all their values. The solutions for (1.3) and (1.4) have been determined using Newton's method. Table 1 lists some values of $\gamma_{n}^{*}$ and $\hat{\gamma}_{n}$, and for the case (4a) the relevant values of the double roots $x_{n}^{*}$. Tables 2 and 3 give the values of the first twelve roots of (4a) and (4b), for some values of $\gamma$, respectively. The numerical solutions have been obtained by a $370 / 168$ IBM computer, using the iterative method developed by Ward [7] and modified by Bach [8]. The computed roots have been used in [2], [5] and, in the hypothesis that the boundary conditions are sufficiently smooth, give a good convergence to the data for the PF series solution of Eq. (3).

Department of Mathematics and Physics

University of Camerino

62032 Camerino, Italy

1. D. D. JOSEPH, "The convergence of biorthogonal series for biharmonic Stokes flow edge problem. I," SIAM J. Appl. Math., v. 33, 1977, pp. 337-347.

2. L. M. DE Socio \& L. Misici, "Convezione in un mezzo poroso causata da sorgenti di calore," Aereotecnica Missili e Spazio, v. 60, no. 4, 1980, pp. 201-206.

3. H. C. Brinkman, "A calculation of the viscous force exerted by a flowing fluid on a dense swarm of particles,” Appl. Sci. Res., v. Al, 1947, pp. 27-34.

4. H. C. BRinkman, "On the permeability of media consisting of closely packed porous particles," Appl. Sci. Res., v. A1, 1947, pp. 81-86.

5. L. M. DE Socio, G. Gaffuri \& L. Misici, "Stokes flow in a rectangular well. Natural convection and boundary layer function," Quart. Appl. Math., v. 39, 1982, pp. 499-508.

6. G. H. HARDY, "On the zeros of the integral function $x-\sin x$," Messenger Math. n.s., v. 31, 1902, pp. $161-165$.

7. J. A. WARD, “The downhill method of solving $f(z)=0$," J. Assoc. Comput. Mach., v. 4, 1957, pp. $148-150$.

8. J. A. BACH, “On the downhill method," Comm. ACM, v. 12, 1969, pp. 675-677. 